\title{
Approximate forms of the pair-density-functional kinetic energy on the basis of a rigorous expression with coupling-constant integration
}

\author{
Katsuhiko Higuchi \\ Graduate School of Advanced Sciences of Matter, Hiroshima University, Higashi-Hiroshima 739-8527, Japan \\ Masahiko Higuchi \\ Department of Physics, Faculty of Science, Shinshu University, Matsumoto 390-8621, Japan \\ (Received 27 January 2014; revised manuscript received 27 October 2014; published 15 December 2014)
}

\begin{abstract}
We propose approximate kinetic energy (KE) functionals of the pair-density (PD)-functional theory on the basis of the rigorous expression with the coupling-constant integration (RECCI) that has been recently derived [Phys. Rev. A 85, 062508 (2012)]. These approximate functionals consist of the noninteracting KE and correlation energy terms. It is found that the Thomas-Fermi-Weizsäcker functional is shown to be better as the noninteracting KE term than the Thomas-Fermi and Gaussian model functionals. It is also shown that the correlation energy term is also indispensable for the reduction of the KE error, i.e., reductions of both inappropriateness of the approximate functional and error of the resultant PD. Concerning the correlation energy term, we further propose an approximate functional in addition to using the existing familiar functionals. This functional satisfies the scaling property of the KE functional, and yields a reasonable PD in a sense that the KE, electron-electron interaction, and potentials energies tend to be improved with satisfying the virial theorem. The present results not only suggest the usefulness of the RECCI but also provide the guideline for the further improvement of the RECCI-based KE functional.
\end{abstract}

DOI: 10.1103/PhysRevA.90.062511

PACS number(s): 31.15.ec, 31.15.ve, 31.15.xt

\section{INTRODUCTION}

The diagonal element of the second-order reduced density matrix, which is called the pair density (PD), has more information about the electron correlation than the electron density [1-4]. For example, the electron density, exchange-correlation (xc) energy, and xc hole can be exactly calculated from the PD. Therefore, the reproduction of the PD is an interesting subject in the field of the development of the first-principles theory. For this aim, the PD functional theory, which was proposed by Ziesche [5,6], provides a powerful method to calculate the ground-state PD [7-26]. In addition to the reproduction of the ground-state PD, the advantage of the PD functional theory over the conventional density-functional theory (DFT) $[27,28]$ is that we do not need the approximation of the xc energy functional because it can be expressed rigorously in terms of the PD [1-4]. Namely, in the conventional DFT both xc energy and kinetic energy (KE) should be approximated appropriately while only the approximate form of the $\mathrm{KE}$ is needed in the PD functional theory.

In order to perform actual calculations on the basis of the PD functional theory, there are two problems that should be overcome. One is how the variational principle with respect to the PD is performed with keeping the search region of PDs within the set of $N$-representable PDs [1-4,29-40]. Another problem is that the approximate form of the KE functional has to be developed in a form of the PD functional, as mentioned above [41-45]. These two problems should be solved simultaneously.

Concerning the first problem, there are a lot of works where the necessary and sufficient conditions for the $N$ representability of the PD are discussed [1-4,29-40]. However, the necessary and sufficient conditions are not yet known in a practical form [1-4,29-40]. In order to avoid this problem, we have proposed several schemes so far [18-20,22-25]. Most recently, we have developed the $\mathrm{PD}$ functional theory utilizing the electron coordinates scaling of PDs [24,25]. This method can substantially extend the search region of PDs without additional heavy tasks of calculations, and is called the "scaling method" [24,25]. The scaling method is employed also in this paper when we check the validity of approximate KE functionals (Sec. IV).

Concerning the second problem, we can consult the way to develop the xc energy functional of the conventional DFT and current-density-functional theory (CDFT) [46-49]. Namely, there are two schemes for developing approximate forms of the energy functional [45]. One is to employ as restrictive conditions exact relations and bounds that should be satisfied by the energy functional. This scheme is hereafter referred as scheme A. This scheme A has been actually used in developing the generalized gradient approximation [50-53], densitymoment approximation [54-60] of the xc energy functional of the DFT, and vorticity expansion approximation [61-64] of the xc energy functional of the CDFT [46-49]. Along scheme A, we have already started to develop the approximate $\mathrm{KE}$ functional [18-20,22-25]. Several approximate forms have been obtained by using exact relations that are derived from the scaling property of the KE functional and Hohenberg-Kohn theorem of the PD functional theory [18-20,22-25].

Another scheme is to develop approximate forms of the energy functional on the basis of the rigorous expression with the coupling-constant integration (RECCI) [45]. This scheme is hereafter called scheme B. This scheme B has also been used in developing the local density approximation (LDA) [28], average-density approximation [65-68], and weighted-density approximation [66-70] of the xc energy functional of the DFT. Also in the CDFT, we have developed the RECCI-based LDA, ADA, and WDA [71]. By reference to these successful examples, scheme $B$ is expected to be an effective way 
to develop the approximate $\mathrm{KE}$ functional also in the $\mathrm{PD}$ functional theory.

Although we have already derived the RECCI for the $\mathrm{KE}$ functional [45], the specific approximate forms are not yet presented on the basis of it. In this paper, we propose approximate forms of the KE functional along scheme $\mathrm{B}$ and check the validity of their approximate forms by performing atomic structure calculations. We also discuss the usefulness and possibility of scheme B as an alternative way to develop the approximate form of the KE functional.

Organization of this paper is as follows. In Sec. II, we review the RECCI for the KE functional of the PD functional theory. On the basis of it, we present six kinds of approximate KE functionals by using familiar energy functionals. We apply them to atomic structure calculations for $\mathrm{Be}, \mathrm{Ne}$, and $\mathrm{Mg}$ atoms, and perform the error analysis of the $\mathrm{KE}$ and resultant PD. The atomic structure calculations are based on the scaling method of the PD functional theory [24,25]. In Sec. III, we explain the details of the calculation method. In Sec. IV, we present results of the error analysis and discuss the validity of these approximate forms in order to get the guideline for improvements of the RECCI-based KE functional. On the basis of the discussion, in Sec. V, we devise the RECCI-based $\mathrm{KE}$ functional that is consistent with the scaling property of the KE functional. In addition, the validity and soundness of the proposed KE functional is confirmed by atomic structure calculations in Sec. V. Finally, the concluding remarks are given in Sec. VI.

\section{APPROXIMATE KE FUNCTIONAL BASED ON THE RECCI}

\section{A. RECCI for the KE functional}

In this section, we briefly review the RECCI for the KE functional [45] for the convenience of later discussions. The RECCI is derived by considering the Hamiltonian that depends on the coupling constant $\lambda$ :

$$
\hat{H}_{\lambda}=\hat{T}+\hat{V}_{\mathrm{eff}}^{\mathrm{int}}+\hat{V}+\lambda\left(\hat{W}-\hat{V}_{\mathrm{eff}}^{\mathrm{int}}\right),
$$

where $\hat{T}, \hat{W}$, and $\hat{V}$ are operators of the KE, electron-electron interaction energy, and external potential energy, respectively, and where $\hat{V}_{\text {eff }}^{\text {int }}$ denotes the effective potential operator of the electron-electron interaction of the noninteracting reference system [18,20]. It should be noted that Eq. (1) becomes the Hamiltonian of the noninteracting reference system $[18,20]$ and that of the real system when $\lambda=0$ and $\lambda=1$, respectively. If we denote the ground-state wave function of Eq. (1) by $\Psi_{\lambda}$, then the ground-state wave functions of the noninteracting reference system and real system are written by $\Psi_{0}$ and $\Psi_{1}$, respectively. Due to the Hohenberg-Kohn theorem with respect to the PD, $\Psi_{\lambda}$ is the functional of the PD [45]. Therefore, the expectation values of $\hat{T}$ and $\hat{W}$ with respect to $\Psi_{1}$ are expressed as the functional of the ground-state PD $\gamma^{(2)}$ of the real system. If we denote their expectation values as $T\left[\gamma^{(2)}\right]$ and $W\left[\gamma^{(2)}\right]$, then the RECCI for $T\left[\gamma^{(2)}\right]$ is given by [45]

$$
\begin{aligned}
T\left[\gamma^{(2)}\right]= & T_{S}\left[\gamma_{\mathrm{SD}}^{(2)}\left[\gamma^{(2)}\right]\right]+\int_{0}^{1}\left\langle\Psi_{\lambda}\left|\hat{W}-\hat{V}_{\mathrm{eff}}^{\mathrm{int}}\right| \Psi_{\lambda}\right\rangle_{\Omega} d \lambda \\
& -W\left[\gamma^{(2)}\right]+V_{\mathrm{eff}}^{\mathrm{int}}\left[\gamma_{\mathrm{SD}}^{(2)}\left[\gamma^{(2)}\right]\right]
\end{aligned}
$$

where $\gamma_{\mathrm{SD}}^{(2)}\left[\gamma^{(2)}\right]$ denotes the variationally best PD among the set of PDs that are constructed from the Slater determinant (SD), and where $T_{s}\left[\gamma_{\mathrm{SD}}^{(2)}\left[\gamma^{(2)}\right]\right]$ and $V_{\mathrm{eff}}^{\text {int }}\left[\gamma_{\mathrm{SD}}^{(2)}\left[\gamma^{(2)}\right]\right]$ are expectation values of $\hat{T}$ and $\hat{V}_{\text {eff }}^{\text {int }}$ with respect to $\Psi_{0}$. The reason why $\gamma_{\mathrm{SD}}^{(2)}$ is a functional of $\gamma^{(2)}$ is that $\gamma_{\mathrm{SD}}^{(2)}$ is uniquely determined as the approximate PD for the real system whose external potential has one-to-one correspondence to $\gamma^{(2)}$ due to the Hohenberg-Kohn theorem [45]. Although the RECCI Eq. (2) seems to be complicated, this expression really gives a good starting point of developing the approximate form as shown in subsequent sections.

\section{B. RECCI-based approximate KE functionals constructed from familiar energy functionals}

In order to develop the approximate form of $T\left[\gamma^{(2)}\right]$ by means of Eq. (2), we employ the perturbation theory for approximating $\Psi_{\lambda}$ [45]. Specifically, $\hat{T}+\hat{V}_{\mathrm{eff}}^{\text {int }}+\hat{V}$ and $\lambda\left(\hat{W}-\hat{V}_{\text {eff }}^{\text {int }}\right)$ in Eq. (1) are treated as the nonperturbative Hamiltonian and perturbation, respectively. As the lowestorder approximation, by neglecting the second- and higherorder terms of the perturbation series, Eq. (2) is approximated to [45]

$$
T\left[\gamma^{(2)}\right] \approx T_{s}\left[\gamma_{\mathrm{SD}}^{(2)}\left[\gamma^{(2)}\right]\right]-\left(W\left[\gamma^{(2)}\right]-\left\langle\Psi_{0}|\hat{W}| \Psi_{0}\right\rangle\right) .
$$

By using Eq. (3), we can devise the approximate form of the KE functional. The first term of the right-hand side of Eq. (3), $T_{s}\left[\gamma_{\mathrm{SD}}^{(2)}\left[\gamma^{(2)}\right]\right]$, is the KE of the noninteracting system. We immediately come up with the idea of using the model functional for the noninteracting $\mathrm{KE}$ as the approximation of $T_{s}\left[\gamma_{\mathrm{SD}}^{(2)}\left[\gamma^{(2)}\right]\right]$. For instance, the Thomas-Fermi (TF) functional $T_{\mathrm{TF}}[\rho]$ [72-74], Gaussian model functional $T_{\mathrm{GM}}[\rho][74,75]$, and Thomas-Fermi-Weizsäcker (TFW) functional $T_{\mathrm{TFW}}[\rho]$ $[74,76]$ may be used as the approximation of $T_{s}\left[\gamma_{\mathrm{SD}}^{(2)}\left[\gamma^{(2)}\right]\right]$. These are provided as the functional of the electron density $\rho(\mathbf{r})$, which are exactly calculated from the PD. The explicit forms are given by

$$
\begin{gathered}
T_{\mathrm{TF}}[\rho]=\frac{3}{5}(3 \pi)^{2 / 3} \int \rho(\mathbf{r})^{5 / 3} d^{3} r \\
T_{\mathrm{GM}}[\rho]=\frac{3 \pi}{2^{2 / 3}} \int \rho(\mathbf{r})^{5 / 3} d^{3} r \\
T_{\mathrm{TFW}}[\rho]=T_{\mathrm{TF}}[\rho]+\frac{1}{9} T_{W}[\rho],
\end{gathered}
$$

with

$$
\begin{gathered}
T_{W}[\rho]=\frac{1}{8} \int \frac{|\nabla \rho(\mathbf{r})|^{2}}{\rho(\mathbf{r})} d^{3} r, \\
\rho(\mathbf{r})=\frac{2}{N-1} \int \gamma^{(2)}\left(\mathbf{r} \mathbf{r}^{\prime} ; \mathbf{r r}^{\prime}\right) d^{3} r^{\prime} .
\end{gathered}
$$

Next, concerning the second term of the right-hand side of Eq. (3), $W\left[\gamma^{(2)}\right]-\left\langle\Psi_{0}|\hat{W}| \Psi_{0}\right\rangle$ is expected to be comparable in magnitude to the correlation energy because $\Psi_{0}$ is the ground-state wave function of the noninteracting reference system. Therefore, the second term may be approximated by the known functional of the correlation energy $E_{c}[\rho]$. In this paper, we adopt the LDA $E_{c}^{\mathrm{LDA}}[\rho][77]$ and 
Perdew-Burke-Ernzerhof (PBE) functional $E_{c}^{\mathrm{PBE}}[\rho][52]$ as $E_{c}[\rho]$. Thus, we obtain six kinds of approximate forms:

$$
\begin{gathered}
T_{\mathrm{TF}-\mathrm{LDA}}\left[\gamma^{(2)}\right]=T_{\mathrm{TF}}[\rho]-E_{c}^{\mathrm{LDA}}[\rho], \\
T_{\mathrm{TF}-\mathrm{PBE}}\left[\gamma^{(2)}\right]=T_{\mathrm{TF}}[\rho]-E_{c}^{\mathrm{PBE}}[\rho], \\
T_{\mathrm{GM}-\mathrm{LDA}}\left[\gamma^{(2)}\right]=T_{\mathrm{GM}}[\rho]-E_{c}^{\mathrm{LDA}}[\rho], \\
T_{\mathrm{GM}-\mathrm{PBE}\left[\gamma^{(2)}\right]}=T_{\mathrm{GM}}[\rho]-E_{c}^{\mathrm{PBE}}[\rho], \\
T_{\mathrm{TFW}-\mathrm{LDA}}\left[\gamma^{(2)}\right]=T_{\mathrm{TFW}}[\rho]-E_{c}^{\mathrm{LDA}}[\rho], \\
T_{\mathrm{TFW}-\mathrm{PBE}}\left[\gamma^{(2)}\right]=T_{\mathrm{TFW}}[\rho]-E_{c}^{\mathrm{PBE}}[\rho] .
\end{gathered}
$$

Equations (9)-(14) are referred to as the TF-LDA, TF-PBE, GM-LDA, GM-PBE, TFW-LDA, and TFW-PBE functional, respectively. These approximate forms seem to be quite plausible because the similar approximate formula is found in the conventional DFT [78]. No adjustable parameters are included in these KE functionals. This is a desirable feature for evaluating the real value of the RECCI-based KE functional. Thus, the RECCI Eq. (2) provides a solid basis for this type of the approximate form in the PD functional theory.

\section{CALCULATION METHOD}

In order to check the validity of six approximate functionals, and to get the guideline for improvements of the RECCI-based $\mathrm{KE}$ functional, atomic structures of neutral $\mathrm{Be}, \mathrm{Ne}$, and $\mathrm{Mg}$ atoms are calculated by using the scaling method that is a recently proposed method and makes the search region of PDs extended efficiently [24,25]. For comparison, we also perform numerical calculations for $\mathrm{Be}, \mathrm{Ne}$, and $\mathrm{Mg}$ atoms by using $T_{\mathrm{TF}}[\rho]$ alone, $T_{\mathrm{GM}}[\rho]$ alone, and $T_{\mathrm{TFW}}[\rho]$ alone as the approximate form of $T\left[\gamma^{(2)}\right]$. The approximate KE functionals used in the test calculations, which are denoted as $\tilde{T}\left[\gamma^{(2)}\right]$, are summarized in Table I. In order to clarify the combination of the $T_{s}$ part and the $E_{c}$ part in $\tilde{T}\left[\gamma^{(2)}\right]$, we also show both $T_{s}[\rho]$ and $E_{c}[\rho]$ together with $\tilde{T}\left[\gamma^{(2)}\right]$ in Table I. It should be noted that the TFW $E_{c}^{\text {scale }}$, which is listed in the last row of Table I, will be explained in Sec. V as the RECCI-based KE functional that satisfies the scaling property of the KE functional.

TABLE I. The approximate KE functionals $\tilde{T}\left[\gamma^{(2)}\right]$, and their constituent functionals.

\begin{tabular}{llrr}
\hline \hline & $\tilde{T}\left[\gamma^{(2)}\right]$ & $T_{s}$ part & $E_{c}$ part \\
\hline TF & Eq. (4) & $T_{\mathrm{TF}}[\rho]$ & \\
GM & Eq. (5) & $T_{\mathrm{GM}}[\rho]$ & \\
TFW & Eq. (6) & $T_{\mathrm{TFW}}[\rho]$ & \\
TF-LDA & Eq. (9) & $T_{\mathrm{TF}}[\rho]$ & $E_{c}^{\mathrm{LDA}}[\rho]$ \\
GM-LDA & Eq. $(11)$ & $T_{\mathrm{GM}}[\rho]$ & $E_{c}^{\mathrm{LDA}}[\rho]$ \\
TFW-LDA & Eq. (13) & $T_{\mathrm{TFW}}[\rho]$ & $E_{c}^{\mathrm{LDA}}[\rho]$ \\
TF-PBE & Eq. $(10)$ & $T_{\mathrm{TF}}[\rho]$ & $E_{c}^{\mathrm{PBE}}[\rho]$ \\
GM-PBE & Eq. $(12)$ & $T_{\mathrm{GM}}[\rho]$ & $E_{c}^{\mathrm{PBE}}[\rho]$ \\
TFW-PBE & Eq. $(14)$ & $T_{\mathrm{TFW}}[\rho]$ & $E_{c}^{\mathrm{PBE}}[\rho]$ \\
TFW- $E_{c}^{\text {scale }}$ & Eq. $(26)$ & $T_{\mathrm{TFW}}[\rho]$ & $E_{c}^{\mathrm{scale}}\left[\gamma^{(2)}\right]$ \\
\hline \hline
\end{tabular}

The first step of the scaling method is to prepare the seed $P D$ that corresponds to the variationally best $\mathrm{PD}$ among the predetermined search region that is not yet extended by the scaling method [24,25]. In the test calculations, we employ as the predetermined search region the set of PDs that are constructed from the linear combination of SDs [23]. The numbers of SDs used in the test calculations are 345, 1342, and 1627 for $\mathrm{Be}, \mathrm{Ne}$, and $\mathrm{Mg}$, respectively. After getting the seed $\mathrm{PD}$, the search region of PDs is extended by adding scaled PDs to elements of the search region, where scaled PDs are obtained by transforming the seed PD via the uniform scaling of electron coordinates. Next, the corrected PD, which corresponds to the variationally best PD among the extended search region of PDs, is easily obtained along the calculation procedure of the scaling method $[24,25]$. Using the corrected PD, the KE $(T)$, electron-electron interaction energy $(W)$, potential energy $(V)$, and total energy $(E)$ are calculated for $\mathrm{Be}, \mathrm{Ne}$, and $\mathrm{Mg}$ atoms. We also evaluate errors of the KE, electron-electron interaction energy and potential energy by comparing with the results of the $1 / Z$ expansion method [79]. Here, the $1 / Z$ expansion method is a sophisticated calculation method for the atomic structure, which is based on data of configuration interaction (CI) calculations and experiments $[80,81]$.

\section{ERROR ANALYSIS BY MEANS OF THE RECCI-BASED KE FUNCTIONAL CONSTRUCTED FROM FAMILIAR ENERGY FUNCTIONALS}

In this section, the validity of approximate forms is discussed on the basis of calculation results for $\mathrm{Be}, \mathrm{Ne}$, and $\mathrm{Mg}$ atoms. The KE error, especially causes of the KE error, are analyzed and discussed in Sec. IV A.

In Sec. IV B, for the purpose of evaluating the resultant $\mathrm{PD}$, the errors of the potential energy and electron-electron interaction energy are investigated. The xc hole is also calculated from the resultant PD and is compared with the correct reference data obtained from the CI method. Through these analyses, we discuss the guideline for improvements of the RECCI-based KE functional.

\section{A. Kinetic energy}

If the exact and approximate KE functionals are denoted by $T\left[\gamma^{(2)}\right]$ and $\tilde{T}\left[\gamma^{(2)}\right]$, respectively, then the error of the $\mathrm{KE}$ $(\Delta T)$ is generally written as

$$
\Delta T=\tilde{T}\left[\tilde{\gamma}_{0}^{(2)}\right]-T\left[\gamma_{g s}^{(2)}\right]
$$

where $\gamma_{g s}^{(2)}$ is the correct ground-state PD, and where $\tilde{\gamma}_{0}^{(2)}$ denotes the resultant PD that is obtained as the solution of the scaling method $[24,25]$. The error $\Delta T$ can be divided into two terms:

$$
\Delta T=\left\{\tilde{T}\left[\gamma_{g s}^{(2)}\right]-T\left[\gamma_{g s}^{(2)}\right]\right\}+\left\{\tilde{T}\left[\tilde{\gamma}_{0}^{(2)}\right]-\tilde{T}\left[\gamma_{g s}^{(2)}\right]\right\} .
$$

The first term of the right-hand side of Eq. (16) can be recognized as the error that comes from the inappropriateness of the approximate functional. Namely, this error is observed even though $\gamma_{g s}^{(2)}$ is input into the approximate functional. The second term of the right-hand side of Eq. (16) can be recognized as the error that comes from the difference between 
TABLE II. Calculation results of $\tilde{T}\left[\gamma_{g s}^{(2)}\right], \Delta T_{\text {functional }}$, and $T_{s}$ and $E_{c}$ parts of $\tilde{T}\left[\gamma_{g s}^{(2)}\right]$ for the Ne atom. When estimating $\Delta T_{\text {functional }}$, we also refer to the result of the $1 / Z$ expansion method [79] as the correct $\operatorname{KE} T\left[\gamma_{g s}^{(2)}\right]$.

\begin{tabular}{lccccc}
\hline \hline & $\begin{array}{c}\tilde{T}\left[\gamma_{g s}^{(2)}\right] \\
(\mathrm{Ry})\end{array}$ & $\begin{array}{c}\Delta T_{\text {functional }} \\
(\mathrm{Ry})\end{array}$ & $\begin{array}{c}\Delta T_{\text {functional }} \\
(\%)\end{array}$ & $\begin{array}{c}T_{s} \text { part } \\
(\mathrm{Ry})\end{array}$ & $\begin{array}{c}E_{c} \text { part } \\
(\mathrm{Ry})\end{array}$ \\
\hline TF & 235.502 & -22.372 & -8.675 & 235.502 & 0.000 \\
GM & 243.490 & -14.384 & -5.578 & 243.490 & 0.000 \\
TFW & 255.616 & -2.258 & -0.876 & 255.616 & 0.000 \\
TF-LDA & 237.334 & -20.540 & -7.965 & 235.502 & -1.832 \\
GM-LDA & 245.322 & -12.552 & -4.868 & 243.490 & -1.832 \\
TFW-LDA & 257.447 & -0.427 & -0.165 & 255.616 & -1.832 \\
TF-PBE & 236.201 & -21.673 & -8.404 & 235.502 & -0.699 \\
GM-PBE & 244.189 & -13.685 & -5.307 & 243.490 & -0.699 \\
TFW-PBE & 256.315 & -1.559 & -0.605 & 255.616 & -0.699 \\
TFW- $E_{c}^{\text {scale }}$ & 256.931 & -0.942 & -0.365 & 255.616 & -1.316 \\
\hline \hline
\end{tabular}

$\tilde{\gamma}_{0}^{(2)}$ and $\gamma_{g s}^{(2)}$ [see Eq. (19) shown below]. Let us denote these errors as $\Delta T_{\text {functional }}$ and $\Delta T_{\text {density }}$ :

$$
\begin{aligned}
\Delta T_{\text {functional }} & =\tilde{T}\left[\gamma_{g s}^{(2)}\right]-T\left[\gamma_{g s}^{(2)}\right], \\
\Delta T_{\text {density }} & =\tilde{T}\left[\tilde{\gamma}_{0}^{(2)}\right]-\tilde{T}\left[\gamma_{g s}^{(2)}\right] .
\end{aligned}
$$

It is worthwhile to rewrite $\Delta T_{\text {density }}$ by using the Taylor series expansion of $\tilde{T}\left[\gamma^{(2)}\right]$ around $\gamma^{(2)}=\gamma_{g s}^{(2)}$, i.e.,

$$
\begin{aligned}
\Delta T_{\text {density }}= & \iint\left\{\tilde{\gamma}_{0}^{(2)}\left(\mathbf{r r}^{\prime} ; \mathbf{r r}^{\prime}\right)-\gamma_{g s}^{(2)}\left(\mathbf{r r}^{\prime} ; \mathbf{r r}^{\prime}\right)\right\} \\
& \times\left\{\frac{\delta \tilde{T}\left[\gamma^{(2)}\right]}{\delta \gamma^{(2)}\left(\mathbf{r r}^{\prime} ; \mathbf{r r}\right)}\right\}_{\gamma^{(2)}=\gamma_{g s}^{(2)}} d^{3} r d^{3} r^{\prime}+\cdots
\end{aligned}
$$

It is clear from this equation that $\Delta T_{\text {density }}$ is the error reflecting the difference between $\tilde{\gamma}_{0}^{(2)}$ and $\gamma_{g s}^{(2)}$.

Since the present approximate KE functionals are the functional of the electron density $\rho(\mathbf{r})$, the CI electron density for the $\mathrm{Ne}$ atom [82] can be utilized as the correct ground-state electron density in evaluating $\Delta T_{\text {functional }}$ and $\Delta T_{\text {density }}$.

\section{Evaluation of $\Delta T_{\text {functional }}$ for $\mathrm{Ne}$}

Using the CI electron density [82] as the correct groundstate electron density, we evaluate $\Delta T_{\text {functional }}$ for approximate forms shown in Table I. The results of $\Delta T_{\text {functional }}$ for the Ne atom are summarized in Table II. It is found that resultant $\Delta T_{\text {functional }}$ 's for all approximate forms are negative. This means that the present approximate forms tend to underestimate the KE. In order to investigate in more detail, we show the $T_{s}$ part and the $E_{c}$ part separately in Table II. The underestimation is reduced if the TFW functional is used as the $T_{s}$ part. The underestimation is also reduced by the $E_{c}$ part, i.e., $E_{c}^{\mathrm{LDA}}[\rho]$ and $E_{c}^{\mathrm{PBE}}[\rho]$ decrease $\Delta T_{\text {functional }}$ by about $0.7 \%$ and $0.2 \%$, respectively (Table II). Two parts, the $T_{s}$ part and the $E_{c}$ part, would have the tendency to cancel each other.

The correction by $E_{c}^{\mathrm{LDA}}[\rho]$ is more effective than that by $E_{c}^{\mathrm{PBE}}[\rho]$, which seems to be due to the accidental cancellation of errors between the $T_{s}$ part and the $E_{c}$ part. Namely, the LDA tends to overestimate the correlation energy due to the logarithmic divergence in the high density limit [74]. This overestimation of the correlation energy accidentally reduces the underestimation of the KE well. This means that the improvement of either the $T_{s}$ part or the $E_{c}$ part is not sufficient, and that well-balanced improvement of both parts is indispensable for the reduction of $\Delta T_{\text {functional }}$.

\section{Evaluation of $\Delta T_{\text {density }}$ for $\mathrm{Ne}$}

Evaluation results of $\Delta T_{\text {density }}$ for the $\mathrm{Ne}$ atom are given in Table III. It is found from Table III that $\Delta T_{\text {density's for }}$ all approximate forms are positive. This means that the resultant $\mathrm{PD}$ and the corresponding electron density lead to the overestimation of the KE. It is also found from Table III that the $E_{c}$ part substantially affects the resultant PD, and accordingly $\Delta T_{\text {density }}$ is reduced. Specifically, $E_{c}$ parts of the LDA and PBE reduce $\Delta T_{\text {density }}$ by about $0.5 \mathrm{Ry}$ and $0.3 \mathrm{Ry}$, respectively. Thus, the $E_{c}$ part decreases $\Delta T_{\text {density }}$ reasonably.

Figure 1 shows resultant electron densities that are calculated by using the TF-PBE, GM-PBE, and TFW-PBE functionals, together with the CI electron density. From this figure, the electron density strongly depends on the approximate form of the $T_{s}$ part. The TFW-PBE functional provides the electron density that is closer to the CI electron density than

TABLE III. Calculation results of $\tilde{T}\left[\tilde{\gamma}_{0}^{(2)}\right], \tilde{T}\left[\gamma_{g s}^{(2)}\right], \Delta T_{\text {density }}$, and $T_{s}$ and $E_{c}$ parts of $\tilde{T}\left[\tilde{\gamma}_{0}^{(2)}\right]$ for the Ne atom. $\tilde{T}\left[\gamma_{g s}^{(2)}\right]$ in the second column are identical to those in Table II.

\begin{tabular}{lcccccr}
\hline \hline & $\begin{array}{c}\tilde{T}\left[\tilde{\gamma}_{0}^{(2)}\right] \\
(\mathrm{Ry})\end{array}$ & $\begin{array}{c}\tilde{T}\left[\gamma_{g s}^{(2)}\right] \\
(\mathrm{Ry})\end{array}$ & $\begin{array}{c}\Delta T_{\text {density }} \\
(\mathrm{Ry})\end{array}$ & $\begin{array}{c}\Delta T_{\text {density }} \\
(\%)\end{array}$ & $\begin{array}{c}T_{s} \text { part } \\
(\mathrm{Ry})\end{array}$ & $\begin{array}{r}E_{c} \text { part } \\
(\mathrm{Ry})\end{array}$ \\
\hline TF & 281.085 & 235.502 & 45.582 & 19.355 & 281.085 & 0.000 \\
GM & 272.595 & 243.490 & 29.105 & 11.953 & 272.595 & 0.000 \\
TFW & 259.029 & 255.616 & 3.414 & 1.335 & 259.029 & 0.000 \\
TF-LDA & 282.521 & 237.334 & 45.187 & 19.039 & 280.717 & -1.804 \\
GM-LDA & 273.926 & 245.322 & 28.604 & 11.660 & 272.202 & -1.724 \\
TFW-LDA & 260.277 & 257.447 & 2.830 & 1.099 & 258.545 & -1.733 \\
TF-PBE & 281.538 & 236.201 & 45.337 & 19.194 & 280.920 & -0.618 \\
GM-PBE & 272.989 & 244.189 & 28.800 & 11.794 & 272.401 & -0.588 \\
TFW-PBE & 259.426 & 256.315 & 3.112 & 1.214 & 258.796 & -0.631 \\
TFW- $E_{c}^{\text {scale }}$ & 257.707 & 256.931 & 0.776 & 0.302 & 256.897 & -0.811 \\
\hline \hline
\end{tabular}




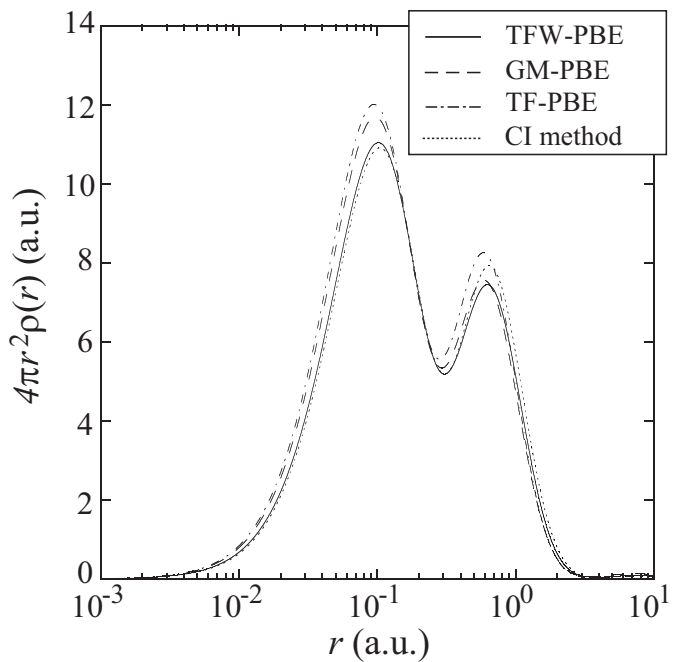

FIG. 1. Profiles of electron densities of the $\mathrm{Ne}$ atom. These profiles are calculated by using the TF-PBE (chain line), GMPBE (dashed line), and TFW-PBE functionals (solid line). As a reference, the electron density by the CI method [82] is also shown (dotted line).

the TF-PBE and GM-PBE functionals. In agreement with this improvement in the electron density, the magnitude of $\Delta T_{\text {density }}$ is also smallest as shown in Table III.

Figures 2-4 show the dependencies of the electron density on the $E_{c}$ part. Although it apparently seems that the $E_{c}$ part has a little effect on the electron density as shown in Figs. 2-4, quantitative differences in $\Delta T_{\text {density }}$ can be found in Table III, and the $E_{c}$ part definitely improves $\Delta T_{\text {density }}$, which is also shown in Table III. This is because $\Delta T_{\text {density }}$ is an integrated value, and because $\Delta T_{\text {density }}$ depends on not only the error of the electron density but also the functional derivative $\left\{\delta \tilde{T}\left[\gamma^{(2)}\right] / \delta \gamma^{(2)}\left(\mathbf{r r}^{\prime} ; \mathbf{r r}^{\prime}\right)\right\}_{\gamma^{(2)}=\gamma_{g s}^{(2)}}$ as shown in Eq. (19).

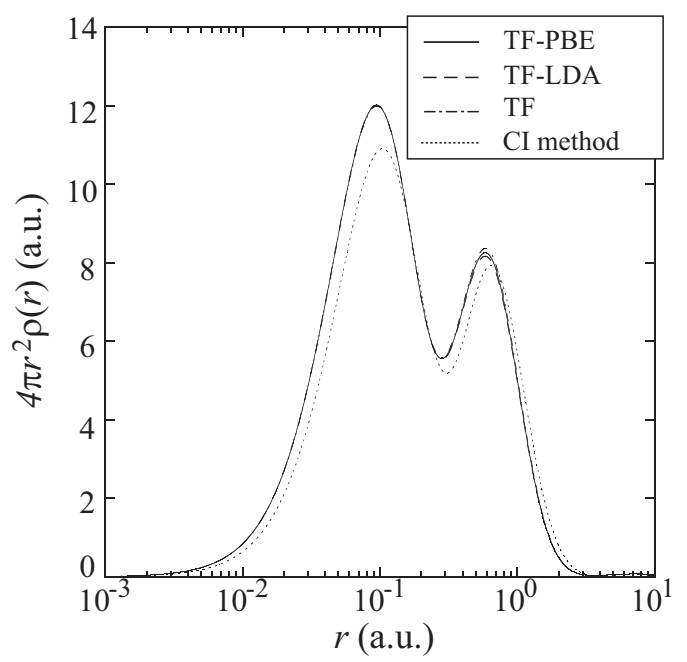

FIG. 2. Profiles of electron densities of the Ne atom. These profiles are calculated by using the TF (chain line), TF-LDA (dashed line), and TF-PBE functionals (solid line). As a reference, the electron density by the CI method [82] is also shown (dotted line).

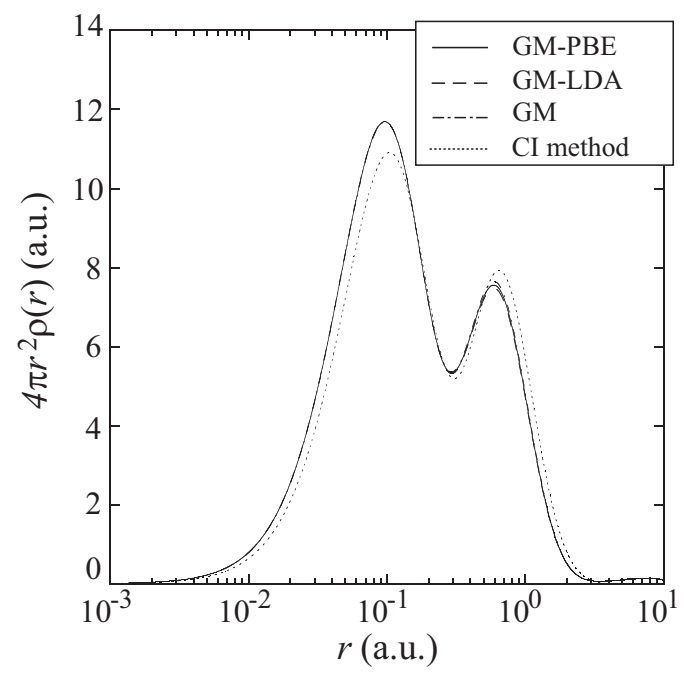

FIG. 3. Profiles of electron densities of the $\mathrm{Ne}$ atom. These profiles are calculated by using the GM (chain line), GM-LDA (dashed line), and GM-PBE functionals (solid line). As a reference, the electron density by the CI method [82] is also shown (dotted line).

\section{Evaluation of $\Delta T$ for $\mathrm{Ne}$, and those for $\mathrm{Be}$ and $\mathrm{Mg}$}

As mentioned in Secs. IV A 1 and IV A 2 , since $\Delta T_{\text {density }}$ has an opposite sign to $\Delta T_{\text {functional }}$, they cancel each other. These cancellations occur in all cases of the present approximate KE functionals, as shown in Table IV. Especially, the resultant total errors $\Delta T$ 's for the TFW-based KE functionals (TFW, TFWLDA, TWF-PBE) are quite small and less than $1 \%$. Judging from this fact, and considering that the present work is the initial attempt for the approximation of Eq. (3), the TFW-based KE functional seems to be tolerable or sound.

Here note that $\Delta T$ for the TFW functional is less than those for the other functionals (TFW-LDA, TFW-PBE). But

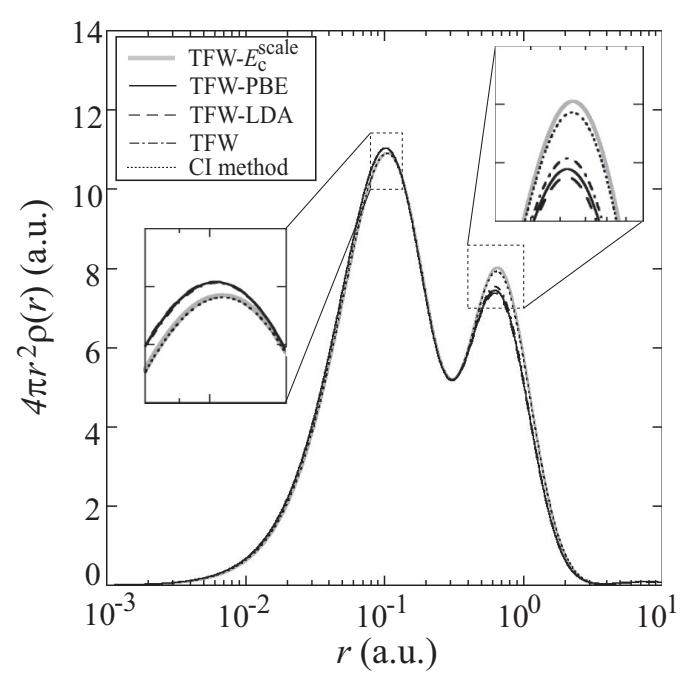

FIG. 4. Profiles of electron densities of the Ne atom. These profiles are calculated by using the TFW (chain line), TFW-LDA (dashed line), TFW-PBE (solid line), and TFW- $E_{c}^{\text {scale }}$ functionals (gray bold line). As a reference, the electron density by the CI method [82] is also shown (dotted line). 
TABLE IV. Calculation results of $\Delta T$ for the Ne atom. For reference, the resultant $\operatorname{KE} \tilde{T}\left[\tilde{\gamma}_{0}^{(2)}\right]$, $\Delta T_{\text {functional }}$, and $\Delta T_{\text {functional }}$ are listed.

\begin{tabular}{lcccccrr}
\hline \hline & $\tilde{T}\left[\tilde{\gamma}_{0}^{(2)}\right]$ & \multicolumn{1}{c}{$\Delta T$} & \multicolumn{1}{c}{$\Delta T$} & $\Delta T_{\text {functional }}$ & \multicolumn{1}{c}{$\Delta T_{\text {functional }}$} & \multicolumn{1}{c}{$\Delta T_{\text {density }}$} & \multicolumn{1}{c}{$\Delta T_{\text {density }}$} \\
& $(\mathrm{Ry})$ & \multicolumn{1}{c}{$(\mathrm{Ry})$} & $(\%)$ & \multicolumn{1}{c}{$(\mathrm{Ry})$} & \multicolumn{1}{c}{$(\mathrm{Ry})$} & $(\%)$ \\
\hline TF & 281.085 & 23.211 & 9.001 & -22.372 & -8.675 & 45.582 & 19.355 \\
GM & 272.595 & 14.721 & 5.709 & -14.384 & -5.578 & 29.105 & 11.953 \\
TFW & 259.029 & 1.155 & 0.448 & -2.258 & -0.876 & 3.414 & 1.335 \\
TF-LDA & 282.521 & 24.647 & 9.558 & -20.540 & -7.965 & 45.187 & 19.039 \\
GM-LDA & 273.926 & 16.052 & 6.225 & -12.552 & -4.868 & 28.604 & 11.660 \\
TFW-LDA & 260.277 & 2.403 & 0.932 & -0.427 & -0.165 & 2.830 & 1.099 \\
TF-PBE & 281.538 & 23.664 & 9.177 & -21.673 & -8.404 & 45.337 & 19.194 \\
GM-PBE & 272.989 & 15.115 & 5.861 & -13.685 & -5.307 & 28.800 & 11.794 \\
TFW-PBE & 259.426 & 1.552 & 0.602 & -1.559 & -0.605 & 3.112 & 1.214 \\
TFW- $E_{c}^{\text {scale }}$ & 257.707 & -0.167 & -0.065 & -0.942 & -0.365 & 0.776 & 0.302 \\
\hline \hline
\end{tabular}

this does not mean that the TFW functional is more reasonable than other approximate forms, because the KE functional can be appreciated as a good approximate form only if both $\Delta T_{\text {density }}$ and $\Delta T_{\text {functional }}$ are improved simultaneously by it. In this sense, the TFW-LDA and TFW-PBE functionals may be regarded as a good approximation as compared with the TFW functional. As shown in Secs. IV A 1 and IV A 2, not only does the $T_{s}$ part explicitly affect the KE error, but also the $E_{c}$ part improves both $\Delta T_{\text {density }}$ and $\Delta T_{\text {functional }}$. The key point to the simultaneous improvements of $\Delta T_{\text {density }}$ and $\Delta T_{\text {functional }}$ is to devise the $T_{s}$ part and the $E_{c}$ part in a well-balanced way. This is an important finding and will be used for the further development of the RECCI-based KE functional (Sec. V).

The calculation results for $\mathrm{Be}$ and $\mathrm{Mg}$ are summarized in Tables V and VI, respectively. Concerning the KE energy, the KE errors for Be and $\mathrm{Mg}$ are reduced by using the TFW functional as the $T_{s}$ part. It is also found in Tables V and VI that $E_{c}$ parts obviously affect $\Delta T$, but sometimes decrease and sometimes increase $\Delta T$, similarly to the case of the $\mathrm{Ne}$ atom (Table IV). This is expected to be because the accidental cancellation of $\Delta T_{\text {density }}$ and $\Delta T_{\text {functional }}$ occurs for the cases of $\mathrm{Be}$ and $\mathrm{Mg}$ atoms as is the same for the $\mathrm{Ne}$ atom.

At the end of this section, we compare the present approximate forms with the previously proposed one that is devised along scheme A [25]. Hereafter, we refer to this functional as the scheme-A KE functional. The errors for the present approximate forms are still large as compared with that

TABLE V. Calculation results of $\Delta T$ and $\tilde{T}\left[\tilde{\gamma}_{0}^{(2)}\right]$ for the Be atom.

\begin{tabular}{lcrr}
\hline \hline & $\tilde{T}\left[\tilde{\gamma}_{0}^{(2)}\right](\mathrm{Ry})$ & $\Delta T(\mathrm{Ry})$ & $\Delta T(\%)$ \\
\hline TF & 32.392 & 3.058 & 10.423 \\
GM & 31.337 & 2.003 & 6.828 \\
TFW & 29.044 & -0.290 & -0.990 \\
TF-LDA & 32.741 & 3.407 & 11.615 \\
GM-LDA & 31.682 & 2.348 & 8.006 \\
TFW-LDA & 29.373 & 0.039 & 0.133 \\
TF-PBE & 32.387 & 3.053 & 10.409 \\
GM-PBE & 31.345 & 2.011 & 6.856 \\
TFW-PBE & 29.074 & -0.260 & -0.887 \\
TFW- $E_{c}^{\text {scale }}$ & 28.816 & -0.518 & -1.765 \\
\hline \hline
\end{tabular}

for the scheme-A KE functional [25]. This means that there is room for improvement in the present approximate forms. However, on the other hand, the present approximate forms (RECCI-based KE functional) never contain parameters, while the scheme-A KE functional contains parameters that should be determined in actual calculations [25]. Therefore, the RECCI-based KE functional is more suitable for measuring the potential of the PD functional theory than the previous one. In addition, the calculation process to determine such parameters is not needed for the present approximate forms. From these points of view, the RECCI-based KE functional seems to be more promising than the scheme-A KE functional for further developments of the KE functional.

\section{B. Potential and electron-electron interaction energies}

\section{Error of the potential energy}

Calculation results of the potential energy for $\mathrm{Ne}, \mathrm{Be}$, and $\mathrm{Mg}$ atoms are given in Tables VII-IX, respectively. We shall at first discuss the results of the Ne atom (Table VII). The error of the potential energy $(\Delta V)$ is reduced by replacing the TF functional with the GM functional or with the TFW functional. The reduction rates for the former and latter replacements are about $4 \%$ and $8 \%$, respectively. This dependence of $\Delta V$ on the $T_{s}$ part is consistent with that of the electron density (Fig. 1). In accordance with the fact that the $E_{c}$ part substantially affects the electron density, which is mentioned in Sec. IV A 2, the

TABLE VI. Calculation results of $\Delta T$ and $\tilde{T}\left[\tilde{\gamma}_{0}^{(2)}\right]$ for the $\mathrm{Mg}$ atom.

\begin{tabular}{lcrr}
\hline \hline & $\tilde{T}\left[\tilde{\gamma}_{0}^{(2)}\right](\mathrm{Ry})$ & $\Delta T(\mathrm{Ry})$ & $\Delta T(\%)$ \\
\hline TF & 433.344 & 33.234 & 8.306 \\
GM & 418.897 & 18.787 & 4.695 \\
TFW & 401.080 & 0.970 & 0.242 \\
TF-LDA & 434.553 & 34.443 & 8.608 \\
GM-LDA & 420.364 & 20.254 & 5.062 \\
TFW-LDA & 402.612 & 2.502 & 0.625 \\
TF-PBE & 433.691 & 33.581 & 8.393 \\
GM-PBE & 419.528 & 19.418 & 4.853 \\
TFW-PBE & 401.945 & 1.835 & 0.459 \\
TFW- $E_{c}^{\text {scale }}$ & 399.919 & -0.191 & -0.048 \\
\hline
\end{tabular}


TABLE VII. Calculation results for the Ne atom. The KE $(T)$, electron-electron interaction energy $(W)$, potential energy $(V)$, and total energy $(E)$ are shown together with their errors to the reference data. The errors are shown in the parentheses. The reference data are also shown in the last row [79].

\begin{tabular}{lrrrr}
\hline \hline & $T(\mathrm{Ry})$ & \multicolumn{1}{c}{$W(\mathrm{Ry})$} & $V(\mathrm{Ry})$ & \multicolumn{1}{c}{$E(\mathrm{Ry})$} \\
\hline TF & 281.085 & 112.837 & -675.006 & -281.085 \\
& $(9.001 \%)$ & $(6.012 \%)$ & $(8.489 \%)$ & $(9.001 \%)$ \\
GM & 272.595 & 102.675 & -647.866 & -272.595 \\
& $(5.709 \%)$ & $(-3.535 \%)$ & $(4.127 \%)$ & $(5.709 \%)$ \\
TFW & 259.029 & 101.601 & -619.660 & -259.029 \\
& $(0.448 \%)$ & $(-4.544 \%)$ & $(-0.406 \%)$ & $(0.448 \%)$ \\
TF-LDA & 282.521 & 110.071 & -672.096 & -279.504 \\
& $(9.558 \%)$ & $(3.413 \%)$ & $(8.022 \%)$ & $(8.388 \%)$ \\
GM-LDA & 273.926 & 100.278 & -645.258 & -271.054 \\
& $(6.225 \%)$ & $(-5.787 \%)$ & $(3.708 \%)$ & $(5.111 \%)$ \\
TFW-LDA & 260.277 & 99.347 & -617.019 & -257.394 \\
& $(0.932 \%)$ & $(-6.662 \%)$ & $(-0.831 \%)$ & $(-0.186 \%)$ \\
TF-PBE & 281.538 & 111.304 & -673.437 & -280.594 \\
& $(9.177 \%)$ & $(4.572 \%)$ & $(8.237 \%)$ & $(8.811 \%)$ \\
GM-PBE & 272.989 & 101.405 & -646.500 & -272.106 \\
& $(5.861 \%)$ & $(-4.728 \%)$ & $(3.908 \%)$ & $(5.519 \%)$ \\
TFW-PBE & 259.426 & 100.334 & -618.216 & -258.455 \\
& $(0.602 \%)$ & $(-5.735 \%)$ & $(-0.638 \%)$ & $(0.225 \%)$ \\
TFW-E ${ }_{c}^{\text {scale }}$ & 257.707 & 108.578 & -623.993 & -257.707 \\
Reference & $(-0.065 \%)$ & $(2.011 \%)$ & $(0.290 \%)$ & $(-0.065 \%)$ \\
data [79] & 257.874 & 106.438 & -622.186 & -257.874 \\
\hline \hline & & & & \\
\hline
\end{tabular}

dependence of $\Delta V$ on the $E_{c}$ part is definitely found in Table VII. This is because $\Delta V$ is also an integrated value similar to the case of $\Delta T_{\text {density }}$.

Similar results of $\Delta V$ 's are obtained for Be and $\mathrm{Mg}$ atoms (Tables VIII and IX). Namely, $\Delta V$ 's are reduced if the TFW functional is used as the $T_{s}$ part instead of using the TF or GM functional. In addition, $\Delta V$ 's are changed obviously by the $E_{c}$ part. These similarities between data of three tables (Tables VII-IX) suggest that the present KE functionals have a kind of universality which is independent of the system.

\section{Error of the electron-electron interaction energy}

Errors of the electron-electron interaction energy $(\Delta W)$ for $\mathrm{Ne}, \mathrm{Be}$, and $\mathrm{Mg}$ atoms are also shown in Tables VII, VIII, and IX, respectively. We discuss $\Delta W$ for the $\mathrm{Ne}$ atom at first (Table VII). It is found in Table VII that if the TF functional is adopted as the $T_{s}$ part, then the electron-electron interaction energy is overestimated. Then, the $E_{c}$ part reduces $\Delta W$ well in this case. On the other hand, the electron-electron interaction energy is underestimated if the GM or TFW functional is used as the $T_{s}$ part. In this case (GM or TFW functional), the $E_{c}$ part slightly enhances $\Delta W$. Although $\Delta W$ is actually one of the useful benchmarks for evaluating the resultant PD, it is difficult in the present case to judge only from values of $\Delta W$ which functional is best for reproducing the ground-state PD.
TABLE VIII. Calculation results for the Be atom. The KE $(T)$, electron-electron interaction energy $(W)$, potential energy $(V)$, and total energy $(E)$ are shown together with their errors to the reference data. The errors are shown in the parentheses. The reference data are also shown in the last row [79].

\begin{tabular}{|c|c|c|c|c|}
\hline & $T$ (Ry) & $W$ (Ry) & $V$ (Ry) & $E$ (Ry) \\
\hline $\mathrm{TF}$ & $\begin{array}{r}32.392 \\
(10.423 \%)\end{array}$ & $\begin{array}{r}9.858 \\
(12.664 \%)\end{array}$ & $\begin{array}{r}-74.641 \\
(10.714 \%)\end{array}$ & $\begin{array}{r}-32.392 \\
(10.423 \%)\end{array}$ \\
\hline GM & $\begin{array}{r}31.337 \\
(6.828 \%)\end{array}$ & $\begin{array}{r}9.513 \\
(8.723 \%)\end{array}$ & $\begin{array}{c}-72.187 \\
(7.074 \%)\end{array}$ & $\begin{array}{c}-31.337 \\
(6.828 \%)\end{array}$ \\
\hline TFW & $\begin{array}{r}29.044 \\
(-0.990 \%)\end{array}$ & $\begin{array}{r}8.807 \\
(0.656 \%)\end{array}$ & $\begin{array}{r}-66.895 \\
(-0.776 \%)\end{array}$ & $\begin{array}{r}-29.044 \\
(-0.990 \%)\end{array}$ \\
\hline TF-LDA & $\begin{array}{r}32.741 \\
(11.615 \%)\end{array}$ & $\begin{array}{r}9.800 \\
(12.001 \%)\end{array}$ & $\begin{array}{r}-74.367 \\
(10.308 \%)\end{array}$ & $\begin{array}{l}-31.826 \\
(8.495 \%)\end{array}$ \\
\hline GM-LDA & $\begin{array}{r}31.682 \\
(8.006 \%)\end{array}$ & $\begin{array}{r}9.447 \\
(7.970 \%)\end{array}$ & $\begin{array}{c}-71.912 \\
(6.666 \%)\end{array}$ & $\begin{array}{l}-30.783 \\
(4.938 \%)\end{array}$ \\
\hline TFW-LDA & $\begin{array}{r}29.373 \\
(0.133 \%)\end{array}$ & $\begin{array}{r}8.739 \\
(-0.122 \%)\end{array}$ & $\begin{array}{r}-66.617 \\
(-1.188 \%)\end{array}$ & $\begin{array}{r}-28.504 \\
(-2.828 \%)\end{array}$ \\
\hline TF-PBE & $\begin{array}{r}32.387 \\
(10.409 \%)\end{array}$ & $\begin{array}{r}9.817 \\
(12.197 \%)\end{array}$ & $\begin{array}{r}-74.442 \\
(10.419 \%)\end{array}$ & $\begin{array}{c}-32.238 \\
(9.899 \%)\end{array}$ \\
\hline GM-PBE & $\begin{array}{r}31.345 \\
(6.856 \%)\end{array}$ & $\begin{array}{r}9.469 \\
(8.217 \%)\end{array}$ & $\begin{array}{c}-71.995 \\
(6.789 \%)\end{array}$ & $\begin{array}{c}-31.181 \\
(6.296 \%)\end{array}$ \\
\hline TFW-PBE & $\begin{array}{r}29.074 \\
(-0.887 \%)\end{array}$ & $\begin{array}{r}8.764 \\
(0.161 \%)\end{array}$ & $\begin{array}{r}-66.714 \\
(-1.044 \%)\end{array}$ & $\begin{array}{r}-28.877 \\
(-1.559 \%)\end{array}$ \\
\hline TFW- $E_{c}^{\text {scale }}$ & $\begin{array}{r}28.816 \\
(-1.765 \%)\end{array}$ & $\begin{array}{r}8.727 \\
(-0.266 \%)\end{array}$ & $\begin{array}{r}-66.359 \\
(-1.570 \%)\end{array}$ & $\begin{array}{r}-28.816 \\
(-1.765 \%)\end{array}$ \\
\hline $\begin{array}{l}\text { Reference } \\
\text { data [79] }\end{array}$ & 29.334 & 8.750 & -67.418 & -29.334 \\
\hline
\end{tabular}

This is because the cancellation of errors of the resultant PD over the spatial coordinates could occur in calculating $\Delta W$, where we note that $\Delta W$ is obtained from the integration of the error of the resultant PD multiplied by the factor $2 /\left|\mathbf{r}-\mathbf{r}^{\prime}\right|$.

In order to evaluate more directly whether the resultant PD is close to the correct ground-state PD or not, the xc hole is investigated for the $\mathrm{Ne}$ atom. The xc hole that can be calculated from the PD reflects the spatial shape of the PD directly. The xc hole $n_{\mathrm{xc}}\left(\mathbf{r}, \mathbf{r}^{\prime}\right)$ is given by

$$
n_{\mathrm{xc}}\left(\mathbf{r}, \mathbf{r}^{\prime}\right)=\frac{2 \gamma^{(2)}\left(\mathbf{r} \mathbf{r}^{\prime} ; \mathbf{r ~ r}^{\prime}\right)-\rho(\mathbf{r}) \rho\left(\mathbf{r}^{\prime}\right)}{\rho(\mathbf{r})} .
$$

Figure 5 shows profiles of the xc hole calculated by using the TF-PBE, GM-PBE, and TFW-PBE functionals, together with the corresponding $\mathrm{xc}$ hole calculated by the $\mathrm{CI}$ method [83]. These profiles are calculated along the $z$ axis under the condition that the reference electron is placed at $z=0.2$. It is found from Fig. 5 that the xc hole by the TFW-PBE functional is close to that by the CI method [83]. Namely, the width of the large hole around the nucleus $\left(z^{\prime}-z=-0.2\right)$, the profile of the valley around $z^{\prime}-z=-0.8$, and the position of the peak around $z^{\prime}-z=-0.5$ for the case of the TFW-PBE functional are in more agreement with the results by the CI method than those by other functionals. Thus, the investigation of the $\mathrm{xc}$ hole reveals that the TFW functional is better as the $T_{s}$ part than the TF or GM functional. 
TABLE IX. Calculation results for the $\mathrm{Mg}$ atom. The KE $(T)$, electron-electron interaction energy $(W)$, potential energy $(V)$, and total energy $(E)$ are shown together with their errors to the reference data. The errors are shown in the parentheses. The reference data are also shown in the last row [79].

\begin{tabular}{|c|c|c|c|c|}
\hline & $T$ (Ry) & $W(\mathrm{Ry})$ & $V$ (Ry) & $E$ (Ry) \\
\hline $\mathrm{TF}$ & $\begin{array}{r}433.344 \\
(8.306 \%)\end{array}$ & $\begin{array}{r}170.034 \\
(7.459 \%)\end{array}$ & $\begin{array}{r}-1036.722 \\
(8.166 \%)\end{array}$ & $\begin{array}{r}-433.344 \\
(8.306 \%)\end{array}$ \\
\hline GM & $\begin{array}{r}418.897 \\
(4.695 \%)\end{array}$ & $\begin{array}{r}166.257 \\
(5.072 \%)\end{array}$ & $\begin{array}{r}-1004.050 \\
(4.757 \%)\end{array}$ & $\begin{array}{r}-418.897 \\
(4.695 \%)\end{array}$ \\
\hline TFW & $\begin{array}{r}401.080 \\
(0.242 \%)\end{array}$ & $\begin{array}{r}159.133 \\
(0.569 \%)\end{array}$ & $\begin{array}{r}-961.293 \\
(0.296 \%)\end{array}$ & $\begin{array}{r}-401.080 \\
(0.242 \%)\end{array}$ \\
\hline TF-LDA & $\begin{array}{r}434.553 \\
(8.608 \%)\end{array}$ & $\begin{array}{r}173.009 \\
(9.339 \%)\end{array}$ & $\begin{array}{r}-1038.353 \\
(8.336 \%)\end{array}$ & $\begin{array}{r}-430.790 \\
(7.668 \%)\end{array}$ \\
\hline GM-LDA & $\begin{array}{r}420.364 \\
(5.062 \%)\end{array}$ & $\begin{array}{r}166.190 \\
(5.029 \%)\end{array}$ & $\begin{array}{r}-1003.217 \\
(4.671 \%)\end{array}$ & $\begin{array}{r}-416.663 \\
(4.137 \%)\end{array}$ \\
\hline TFW-LDA & $\begin{array}{r}402.612 \\
(0.625 \%)\end{array}$ & $\begin{array}{r}157.409 \\
(-0.520 \%)\end{array}$ & $\begin{array}{r}-959.020 \\
(0.059 \%)\end{array}$ & $\begin{array}{r}-398.998 \\
(-0.278 \%)\end{array}$ \\
\hline TF-PBE & $\begin{array}{r}433.691 \\
(8.393 \%)\end{array}$ & $\begin{array}{r}173.147 \\
(9.426 \%)\end{array}$ & $\begin{array}{r}-1039.087 \\
(8.413 \%)\end{array}$ & $\begin{array}{r}-432.249 \\
(8.033 \%)\end{array}$ \\
\hline GM-PBE & $\begin{array}{r}419.528 \\
(4.853 \%)\end{array}$ & $\begin{array}{r}167.067 \\
(5.584 \%)\end{array}$ & $\begin{array}{r}-1004.633 \\
(4.818 \%)\end{array}$ & $\begin{array}{r}-418.037 \\
(4.481 \%)\end{array}$ \\
\hline TFW-PBE & $\begin{array}{r}401.945 \\
(0.459 \%)\end{array}$ & $\begin{array}{r}158.077 \\
(-0.098 \%)\end{array}$ & $\begin{array}{r}-960.448 \\
(0.208 \%)\end{array}$ & $\begin{array}{r}-400.426 \\
(0.079 \%)\end{array}$ \\
\hline TFW- $E_{c}^{\text {scale }}$ & $\begin{array}{r}399.919 \\
(-0.048 \%)\end{array}$ & $\begin{array}{r}160.331 \\
(1.326 \%)\end{array}$ & $\begin{array}{r}-960.169 \\
(0.179 \%)\end{array}$ & $\begin{array}{r}-399.991 \\
(-0.048 \%)\end{array}$ \\
\hline $\begin{array}{l}\text { Reference } \\
\text { data [79] }\end{array}$ & 400.110 & 158.232 & -958.452 & -400.110 \\
\hline
\end{tabular}

Next, we shall consider the effect of the $E_{c}$ part on the resultant PD. Profiles of the xc hole for the cases of the TFW,

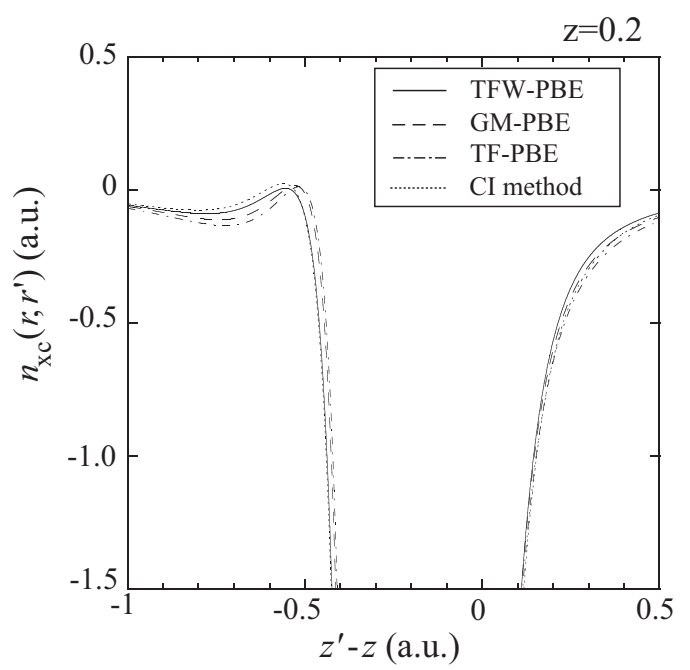

FIG. 5. Profiles of the xc holes $\left[n_{\mathrm{xc}}\left(\mathbf{r}, \mathbf{r}^{\prime}\right)\right]$ of the Ne atom along $z$ axis. The reference electron is placed at $z=0.2$. These profiles are calculated by using the TF-PBE (chain line), GM-PBE (dashed line), and TFW-PBE functionals (solid line). As a reference, the xc hole by the CI method [83] is also shown (dotted line).

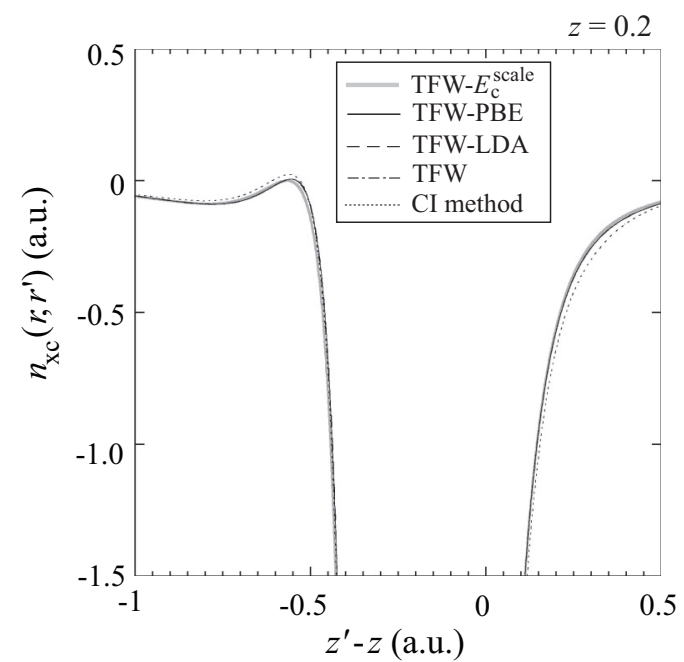

FIG. 6. Profiles of the xc holes $\left[n_{\mathrm{xc}}\left(\mathbf{r}, \mathbf{r}^{\prime}\right)\right]$ of the Ne atom along $z$ axis. The reference electron is placed at $z=0.2$. These profiles are calculated by using the TFW (chain line), TFW-LDA (dashed line), TFW-PBE (solid line) and TFW- $E_{c}^{\text {scale }}$ functionals (gray bold line). As a reference, the xc hole by the CI method [83] is also shown (dotted line).

TFW-LDA, and TFW-PBE functionals are shown in Figs. 6-8 in which the reference electron is placed at $z=0.2$ (Figs. 6 and 7) and $z=0.4$ (Fig. 8), respectively. Although profiles for $z=0.2$ (Figs. 6 and 7) are little dependent on the $E_{c}$ part, profiles for $z=0.4$ (Fig. 8) have some differences between them, especially around $z^{\prime}-z=-0.8$. It is found from Fig. 8 that the $\mathrm{xc}$ hole by the TFW functional is closer to that by the CI method than those by the TFW-LDA and TFW-PBE. This tendency of the $\mathrm{xc}$ hole is consistent with that of $\Delta W$, as shown in Table VII. This tendency of the xc hole is also found

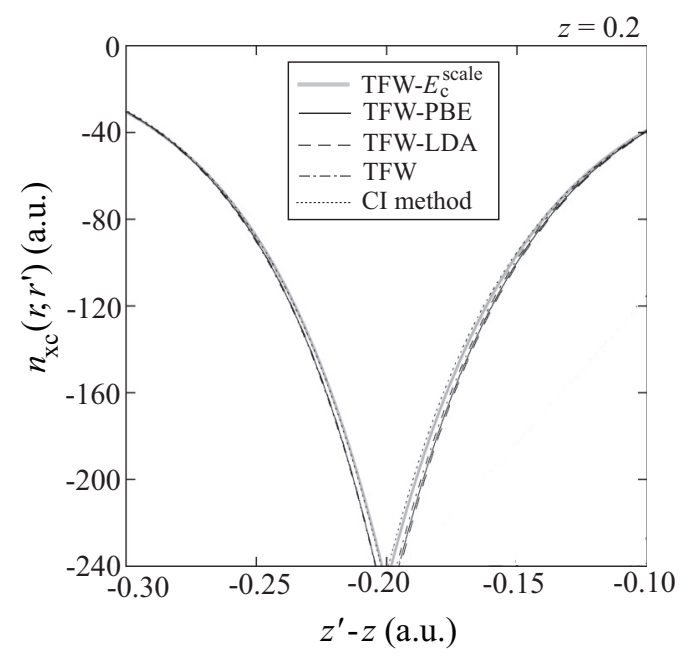

FIG. 7. Profiles of the $\mathrm{xc}$ holes $\left[n_{\mathrm{xc}}\left(\mathbf{r}, \mathbf{r}^{\prime}\right)\right]$ of the Ne atom along $z$ axis. The reference electron is placed at $z=0.2$. These profiles are calculated by using the TFW (chain line), TFW-LDA (dashed line), TFW-PBE (solid line), and TFW- $E_{c}^{\text {scale }}$ functionals (gray bold line). As a reference, the xc hole by the CI method [83] is also shown (dotted line). 


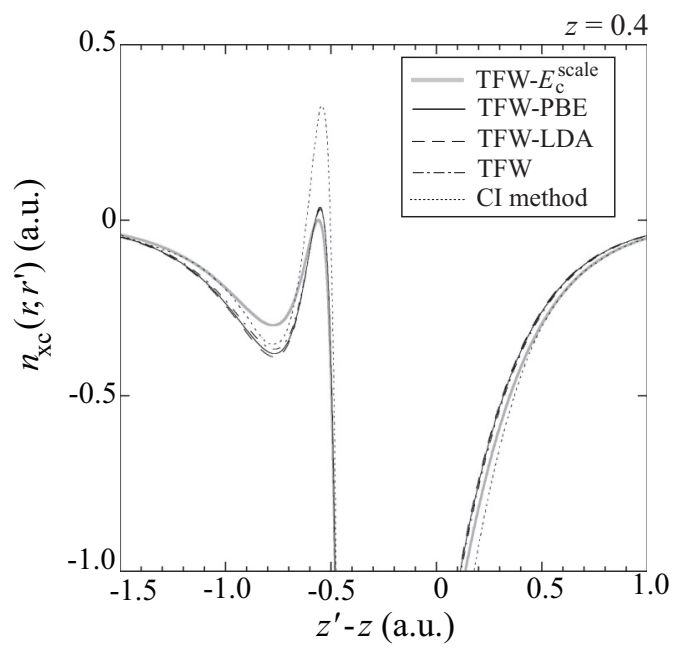

FIG. 8. Profiles of the xc holes $\left[n_{\mathrm{xc}}\left(\mathbf{r}, \mathbf{r}^{\prime}\right)\right]$ of the Ne atom along $z$ axis. The reference electron is placed at $z=0.4$. These profiles are calculated by using the TFW (chain line), TFW-LDA (dashed line), TFW-PBE (solid line), and TFW- $E_{c}^{\text {scale }}$ functionals (gray bold line). As a reference, the xc hole by the CI method [83] is also shown (dotted line).

in the case of using GM functional as the $T_{s}$ part. On the other hand, the xc hole is improved by the $E_{c}$ part in the case of using the TF functional as the $T_{s}$ part, which is also consistent with the results of $\Delta W$ shown in Table VII. Thus, whether the $E_{c}$ part improves the xc hole or not depends on the $T_{s}$ part.

Similar results concerning $\Delta W$ can be found in $\mathrm{Be}$ and $\mathrm{Mg}$ atoms, which are shown in Tables VIII and IX, respectively. It is seen from Tables VIII and IX that $\Delta W$ is reduced by using the TFW as the $T_{s}$ part. This is similar to the tendency of the xc hole of the $\mathrm{Ne}$ atom. The $E_{c}$ part works effectively in reducing $\Delta W$ for the case of the $\mathrm{Be}$ atom, while in the case of the $\mathrm{Mg}$ atom it works effectively only if the TFW is used as the $T_{s}$ part.

Thus, it is shown that although the TFW functional is better as the $T_{s}$ part than the TF or GM functional, the effects of the $E_{c}$ part on $\Delta W$ and the xc hole do not show a clear tendency. Such an absence of the tendency would be caused by the enhancement or cancellation between errors of the resultant PD. Namely, the error of the resultant PD can be divided into two errors that are caused by the $T_{s}$ part and the $E_{c}$ part. These two errors seem to cancel each other, or enhance each other case by case. This means, again, that it is quite significant to develop the $T_{s}$ part and the $E_{c}$ part in a well-balanced way. This will become a useful guideline for the further development of the KE functional.

\section{RECCI-BASED KE FUNCTIONAL CONSISTENT WITH THE SCALING PROPERTY}

With the aim of improving $T_{s}$ and $E_{c}$ parts in a wellbalanced way, we develop the RECCI-based KE functional that is consistent with the scaling property of the KE functional $[9,78,84]$. The solution obtained by the scaling method [24,25] necessarily satisfies the virial theorem if the approximate KE functional is consistent with the scaling property. Furthermore, the scaling property is used as one of the key restrictive conditions also in scheme A [18-20,22-25]. Therefore, it is meaningful to develop the RECCI-based KE functional that has the correct scaling property. In this section, we shall propose such an approximate KE functional and discuss its validity.

\section{A. Approximate form}

In RECCI-based approximate KE functionals discussed in Sec. IV, TF, GM, and TFW functionals that are used as the $T_{s}$ part satisfy the scaling property of the KE functional. Namely, these functionals respectively satisfy the following relations: $T_{\mathrm{TF}}\left[\rho_{\zeta}\right]=\zeta^{2} T_{\mathrm{TF}}[\rho], T_{\mathrm{GM}}\left[\rho_{\zeta}\right]=\zeta^{2} T_{\mathrm{GM}}[\rho]$, $T_{\mathrm{TFW}}\left[\rho_{\zeta}\right]=\zeta^{2} T_{\mathrm{TFW}}[\rho]$, where $\zeta$ is the scaling factor and $\rho_{\zeta}(\mathbf{r})$ denotes the scaled electron density that is given by $\zeta^{3} \rho(\zeta \mathbf{r})$. On the other hand, LDA and PBE functionals that are used as the $E_{c}$ part do not satisfy the scaling property of the KE functional. In order to improve this unbalance, we propose the RECCI-based KE functional in which both the $T_{s}$ part and the $E_{c}$ part are consistent with the scaling property of the KE functional.

Since the TFW functional is better than the TF and GM functionals, as shown in the previous section, we adopt the TFW functional as the $T_{s}$ part. As the $E_{c}$ part, we employ the following functional:

$$
E_{c}^{\text {scale }}\left[\gamma^{(2)}\right]=\alpha[\rho]\left(W\left[\gamma^{(2)}\right]-U[\rho]-E_{x}[\rho]\right),
$$

where $U[\rho]$ is the Hartree energy which can be rigorously expressed by the electron density. The functional $E_{x}[\rho]$ denotes the exchange energy functional. Since the exchange energy cannot be expressed by the PD alone, some approximation is needed. In this paper, we utilize as $E_{x}[\rho]$ the DFT-LDA exchange energy functional that is constructed by borrowing the expression for the exchange energy density of the homogeneous electron liquid [85]. The functional $\alpha[\rho]$ is determined so that $E_{c}^{\text {scale }}\left[\gamma^{(2)}\right]$ satisfies the scaling property of the KE functional: $E_{c}^{\text {scale }}\left[\gamma_{\zeta}^{(2)}\right]=\zeta^{2} E_{c}^{\text {scale }}\left[\gamma^{(2)}\right]$. Namely, if $\alpha[\rho]$ is given in such a way that the relation $\alpha\left[\rho_{\zeta}\right]=\zeta \alpha[\rho]$ holds, then $E_{c}^{\text {scale }}\left[\gamma^{(2)}\right]$ will have the correct scaling property of the KE functional. Here, we use the fact that $W\left[\gamma^{(2)}\right]-U[\rho]-E_{x}[\rho]$ holds the following relation:

$W\left[\gamma_{\zeta}^{(2)}\right]-U\left[\rho_{\zeta}\right]-E_{x}\left[\rho_{\zeta}\right]=\zeta\left(W\left[\gamma^{(2)}\right]-U[\rho]-E_{x}[\rho]\right)$.

In this paper, the following functional is employed as $\alpha[\rho]$ :

$$
\alpha[\rho]=\beta \int \rho(\mathbf{r})^{4 / 3} d^{3} r,
$$

where $\beta$ is a constant. Substituting Eq. (23) into Eq. (21), we have the $E_{c}$ part of the present KE functional:

$$
E_{c}^{\text {scale }}\left[\gamma^{(2)}\right]=\beta \int \rho(\mathbf{r})^{4 / 3} d^{3} r\left(W\left[\gamma^{(2)}\right]-U[\rho]-E_{x}[\rho]\right) .
$$

Next, we shall explain how to determine the value of $\beta$. From Eq. (24), we formally get $\beta=E_{c}^{\text {scale }}\left[\gamma^{(2)}\right] /$ $\int \rho(\mathbf{r})^{4 / 3} d^{3} r\left(W\left[\gamma^{(2)}\right]-U[\rho]-E_{x}[\rho]\right)$. By using the electron density that is obtained through the DFT calculations with the PBE functional [52], we estimate $\int \rho(\mathbf{r})^{4 / 3} d^{3} r, U[\rho]$, 


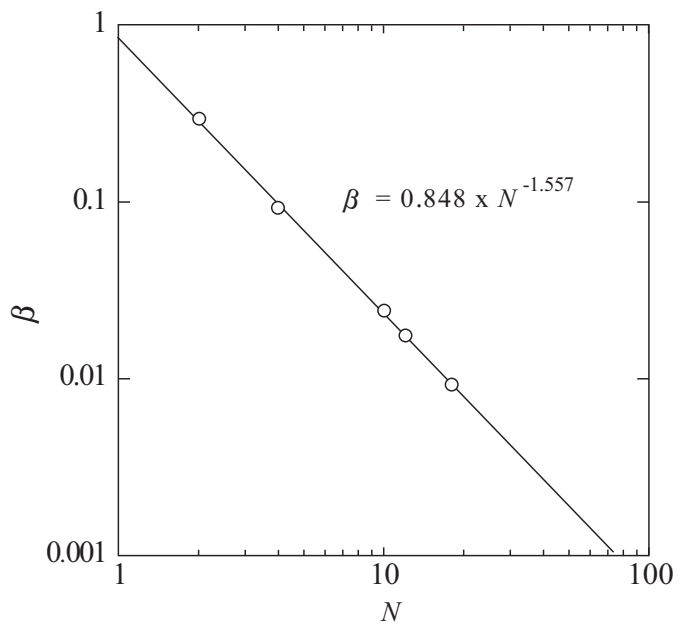

FIG. 9. Dependence of $\beta$ on the total number of electrons $N$.

and $E_{x}[\rho]$. As to $W\left[\gamma^{(2)}\right]$, the results of the $1 / Z$ expansion method [79] is adopted. Also, using the Slater determinant $\Phi_{K S}$ that consists of the Kohn-Sham orbitals, we estimate $E_{c}^{\text {scale }}\left[\gamma^{(2)}\right]$ via $W\left[\gamma^{(2)}\right]-\left\langle\Phi_{K S}|\hat{W}| \Phi_{K S}\right\rangle$. The estimated values of $\beta$ for closed-shell atoms are shown in Fig. 9. From Fig. 9, we have

$$
\beta=0.848 \times N^{-1.557}
$$

to an accuracy with the coefficient of determination $\left(R^{2}\right)$ of 0.992 , where $N$ denotes the total number of electrons. It should be noted that since the variational principle with respect to the PD is performed under the condition that $N$ is unchanged, $\beta$ is regarded as a constant in actual calculations.

We refer to the approximate KE functional with Eq. (24) as $\mathrm{TFW}-E_{c}^{\text {scale }}$, the explicit expression of which is given by

$$
T_{\mathrm{TFW}-\mathrm{E}_{c}^{\text {scale }}}\left[\gamma^{(2)}\right]=T_{\mathrm{TFW}}[\rho]-E_{c}^{\text {scale }}\left[\gamma^{(2)}\right] .
$$

As is clear from the above-mentioned discussion, the TFW$E_{c}^{\text {scale }}$ functional satisfies the correct scaling property of the KE of the PD functional theory $[9,78,84]$. This approximate form does not contain any adjustable parameters that should be determined depending on the system. Therefore, the TFW$E_{c}^{\text {scale }}$ would be applicable not only to atoms and molecules but also solids.

\section{B. Validity check of the TFW- $E_{c}^{\text {scale }}$ functional \\ 1. Error of the $\mathrm{KE}$}

We shall start with the discussion on the error of the KE in the TFW- $E_{c}^{\text {scale }}$ functional. As mentioned in Sec. IV, the error of the KE can be divided into two kinds of errors, $\Delta T_{\text {functional }}$ and $\Delta T_{\text {density }}$. The results of $\Delta T_{\text {functional }}$ and $\Delta T_{\text {density }}$ for Ne are shown in Tables II and III, respectively. It is found that both $\Delta T_{\text {functional }}$ and $\Delta T_{\text {density }}$ are improved by using the TFW- $E_{c}^{\text {scale }}$ functional instead of other KE functionals [86]. It is also found from these tables that $\Delta T_{\text {functional }}$ and $\Delta T_{\text {density }}$ for the TFW- $E_{c}^{\text {scale }}$ functional are negative and positive, respectively, which is similar to the cases of other approximate forms (Table IV). Due to the cancellation of these two reduced errors, $\Delta T$ for $\mathrm{Ne}$ is as small as $-0.119 \%$. This value is much smaller than errors by other approximate KE functional as shown in
Table IV. Also in the case of $\mathrm{Mg}$, the TFW- $E_{c}^{\text {scale }}$ functional leads to the smallest $\Delta T$ (Table VI). On the other hand, in the case of $\mathrm{Be}, \Delta T$ by the TFW- $E_{c}^{\text {scale }}$ functional increases a little as compared to those by the TFW-LDA and TFW-PBE functionals (Table V). This would be because the accidental cancellation between $\Delta T_{\text {functional }}$ and $\Delta T_{\text {density }}$ occurs in cases of using the TFW-LDA and TFW-PBE functionals more effectively than the case of using the TFW- $E_{c}^{\text {scale }}$ functional.

Thus, the TFW- $E_{c}^{\text {scale }}$ functional that is consistent with the scaling property leads to comparable or better KE than the TFW-LDA and TFW-PBE functionals that are not consistent with the scaling property.

\section{Errors of the potential and electron-electron interaction energies}

Calculation results of the potential and electron-electron interaction energies for $\mathrm{Ne}, \mathrm{Be}$, and $\mathrm{Mg}$ atoms are given in Tables VII-IX, respectively. It is found from these tables that the virial theorem $\left(2 T\left[\gamma^{(2)}\right]+W\left[\gamma^{(2)}\right]+V\left[\gamma^{(2)}\right]=0\right)$ $[24,25]$ holds in the case of using the TFW- $E_{c}^{\text {scale }}$ functional, which is confirmed directly by using calculation results of $T$, $W$, and $V$ for $\mathrm{Ne}, \mathrm{Be}$, and $\mathrm{Mg}$ atoms. This is one of the striking features of using the TFW- $E_{c}^{\text {scale }}$ functional.

In order to investigate $\Delta V$ and $\Delta W$, we shall first discuss the results of $\mathrm{Ne}$ (Table VII). As compared to the results by the TFW-LDA and TFW-PBE functionals, $\Delta V$ is much reduced by using the $T F W-E_{c}^{\text {scale }}$ functional. Consistently with this improvement in $\Delta V$, the electron density is also improved as shown in Fig. 4. Both the first and second peaks of the present electron density (gray bold line) are close to the CI electron density as compared with those calculated by other KE functionals.

Similarly to the improvements in $\Delta V$ and $\Delta T$, it is found from Table VII that $\Delta W$ is also improved for Ne by using the TFW- $E_{c}^{\text {scale }}$ functional. As shown in Figs. 6 and 7, the xc hole in the case of $z=0.2$ gets closer to the CI xc hole when the TFW- $E_{c}^{\text {scale }}$ functional is used. Especially, the large hole $\left(z-z^{\prime}=0.2\right)$ that largely contributes to the xc energy $\varepsilon_{\mathrm{xc}}$ is much improved as shown in Fig. 7. On the other hand, in the case of $z=0.4$ (Fig. 8), the xc hole by the TFW- $E_{c}^{\text {scale }}$ functional gets away from the CI xc hole compared with those by other functionals. But, we can say that this discrepancy does not cause a large error in $\varepsilon_{\mathrm{xc}}$. The reason is as follows. The xc energy $\varepsilon_{\mathrm{xc}}$ is written as

$$
\varepsilon_{\mathrm{xc}}=\frac{e^{2}}{2} \iint \frac{\rho(\mathbf{r}) n_{\mathrm{xc}}\left(\mathbf{r}, \mathbf{r}^{\prime}\right)}{\left|\mathbf{r}-\mathbf{r}^{\prime}\right|} d^{3} r d^{3} r^{\prime} .
$$

Equation (27) implies that $n_{\mathrm{xc}}\left(\mathbf{r}, \mathbf{r}^{\prime}\right)$ contributes to $\varepsilon_{\mathrm{xc}}$ largely if the factor $\rho(\mathbf{r}) /\left|\mathbf{r}-\mathbf{r}^{\prime}\right|$ has a large value. Since $\rho(\mathbf{r})$ is large near the nucleus $(\mathbf{r}=0), \rho(\mathbf{r}) /\left|\mathbf{r}-\mathbf{r}^{\prime}\right|$ has a large value at the vicinity of $\mathbf{r}=\mathbf{r}^{\prime}=0$. This means that the accuracy of $n_{\mathrm{xc}}\left(\mathbf{r}, \mathbf{r}^{\prime}\right)$ at $z=0.2$ is more important than that at $z=0.4$ in evaluating $\varepsilon_{\mathrm{xc}}$. Thus, the TFW- $E_{c}^{\text {scale }}$ functional preferentially improves the profile of the $\mathrm{xc}$ hole in the region where the xc hole effectively affects the accuracy of $\varepsilon_{\mathrm{xc}}$. Combining this fact with the improvement in the profile of the electron density (Fig. 4), the improvement of $\Delta W$ in the case of $\mathrm{Ne}$ can be recognized. 
Next, we shall discuss $\Delta V$ and $\Delta W$ for $\mathrm{Be}$ and $\mathrm{Mg}$ atoms (Tables VIII and IX). Concerning $\Delta V^{\prime}$ s for Be and $\mathrm{Mg}$ atoms, there is not a considerable difference between the TFW-LDA, TFW-PBE, and TFW- $E_{c}^{\text {scale }}$ functionals. On the other hand, $\Delta W$ for $\mathrm{Mg}$ worsens a little by using the TFW- $E_{c}^{\text {scale }}$ functional instead of the TFW-LDA or TFW-PBE functional while $\Delta W$ for Be obtained by the TFW- $E_{c}^{\text {scale }}$ functional is also comparable with those by the TFW-LDA and TFW-PBE functionals. This different tendency would be caused by the fact that the scaling method $[24,25]$ with the TFW- $E_{c}^{\text {scale }}$ functional necessarily leads to the solution satisfying the virial theorem. Namely, if any one of $T, W$, and $V$ has a little error, then the others are adjusted so that the virial theorem $2 T+W+V=0$ holds. Thus, different types of cancellations of errors would occur in the case of using the $\mathrm{TFW}-E_{c}^{\text {scale }}$ functional.

Although the degree of improvement of $\Delta V, \Delta W$, and $\Delta T$ have a small variation, the resultant PD would be improved as a whole by using the TFW- $E_{c}^{\text {scale }}$ functional. The key point of the improvement is that the resultant PD obtained by the TFW$E_{c}^{\text {scale }}$ functional satisfies the virial theorem that is regarded as one of the strong necessary conditions for the ground state.

\section{CONCLUDING REMARKS}

Approximate KE functionals of the PD functional theory are proposed on the basis of the RECCI (Table I). The validity and soundness of the approximate KE functionals are checked by applying them to electronic structure calculations for Be, $\mathrm{Ne}$, and $\mathrm{Mg}$ atoms. The striking points of this work are as follows:

(1) All of the approximate forms that are proposed in this work have no adjustable parameters, which enables us to evaluate the real value of scheme B.

(2) According to the error analysis by using the RECCIbased KE functionals that consist of familiar energy functionals (Sec. IV), the $E_{c}$ part plays a non-negligible role in the KE functional, especially it reasonably reduces $\Delta T_{\text {functional }}$ and $\Delta T_{\text {density }}$ for the Ne atom, and also reduces $\Delta W$ and $\Delta V$ for the $\mathrm{Be}, \mathrm{Ne}$, and $\mathrm{Mg}$ atoms.

(3) The matching problem between the $T_{s}$ part and the $E_{c}$ part can be found also from the error analysis. This problem would come from the incompleteness of both the $T_{s}$ part and the $E_{c}$ part. The results of the error analysis suggest that it would be necessary to devise approximate forms of the $T_{s}$ part and the $E_{c}$ part in a well-balanced way.

(4) In order to improve the $T_{s}$ part and the $E_{c}$ part in a well-balanced way, the RECCI-based KE functional that is consistent with the scaling property of the KE functional is proposed (TFW- $E_{c}^{\text {scale }}$ functional). This KE functional successfully leads to the reasonable PD that satisfies the virial theorem. This functional would be the first milestone in developing the approximate $\mathrm{KE}$ functional along scheme $\mathrm{B}$.

Thus, it is shown that the RECCI for the KE functional is a good starting point for developing the approximate form. It would be worthwhile to attempt to add the higher-order correction term [74] into the TFW functional and/or to incorporate the higher-order terms of the perturbation into Eq. (3). When incorporating the higher-order perturbation terms, the correction term will get close to the correct correlation contribution to the KE, i.e., $T_{c}\left(=T-T_{s}\right)$. Many previous works on $T_{c}[55,58,87-89]$ would be helpful in incorporating the higher-order terms. Such attempts and discussions seem to be the next issues.

\section{ACKNOWLEDGMENTS}

This work was partially supported by Grant-in-Aid for Scientific Research (Grants No. 22540390, No. 23540446, No. 26400354, and No. 26400397) of The Ministry of Education, Culture, Sports, Science, and Technology, Japan.
[1] A. J. Coleman, Rev. Mod. Phys. 35, 668 (1963).

[2] Many-Electron Densities and Reduced Density Matrices, edited by J. Closlowski (Kluwer, Dordrecht/Plenum, New York, 2000).

[3] A. J. Coleman and V. I. Yukalov, Reduced Density Matrices: Coulson's Challenge (Springer-Verlag, Berlin, 2000).

[4] The Fundamentals of Electron Density, Density Matrix and Density Functional Theory in Atoms, Molecules and the Solid States, edited by N. I. Gidopoulos and S. Wilson (Kluwer, New York, 2003).

[5] P. Ziesche, Phys. Lett. A 195, 213 (1994).

[6] P. Ziesche, Int. J. Quantum Chem. 60, 1361 (1996).

[7] A. Gonis, T. C. Schulthess, J. van Ek, and P. E. A. Turchi, Phys. Rev. Lett. 77, 2981 (1996).

[8] A. Gonis, T. C. Schulthess, P. E. A. Turchi, and J. van Ek, Phys. Rev. B 56, 9335 (1997).

[9] M. Levy and P. Ziesche, J. Chem. Phys. 115, 9110 (2001).

[10] F. Furche, Phys. Rev. A 70, 022514 (2004).

[11] Á. Nagy, Phys. Rev. A 66, 022505 (2002).

[12] Á. Nagy and C. Amovilli, J. Chem. Phys. 121, 6640 (2004).

[13] B. Hetényi, L. Brualla, and S. Fantoni, Phys. Rev. Lett. 93, 170202 (2004).
[14] J. K. Percus, J. Chem. Phys. 122, 234103 (2005).

[15] P. W. Ayers and M. Levy, J. Chem. Sci. 117, 507 (2005).

[16] Á. Nagy, Int. J. Quantum Chem. 106, 1043 (2006).

[17] P. W. Ayers, S. Golden, and M. Levy, J. Chem. Phys. 124, 054101 (2006).

[18] M. Higuchi and K. Higuchi, Physica B 387, 117 (2007).

[19] M. Higuchi and K. Higuchi, Phys. Rev. A 75, 042510 (2007).

[20] M. Higuchi and K. Higuchi, Phys. Rev. B 78, 125101 (2008).

[21] B. Hetényi and A. W. Hauser, Phys. Rev. B 77, 155110 (2008).

[22] K. Higuchi and M. Higuchi, J. Phys.: Condens. Matter 21, 064206 (2009).

[23] K. Higuchi and M. Higuchi, Phys. Rev. B 82, 155135 (2010).

[24] M. Higuchi and K. Higuchi, Phys. Rev. A 84, 044502 (2011).

[25] K. Higuchi and M. Higuchi, Phys. Rev. A 87, 032511 (2013).

[26] It should be noted that the existence of a minimum point is assumed in the definition of the extended-constrainedsearch-based universal functional of the PD functional theory [5,9-12,18-20,22-25]. The assumption of this type is commonly introduced in the extension theories of the DFT such as the superconductor-DFT, LDA $+U$ method, density-matrix functional theory, current-density functional theory, extended 
constrained-search theory, and so on. But, the mathematical proof of this assumption seems to be not yet completed in these extension theories. It is, of course, significant to prove this assumption mathematically, while it is one way that we apply these theories to actual systems and judge through actual calculations whether the assumption becomes something of a problem or not. Indeed, these empirical approaches have been usually used in the extension theories of the DFT. Also in the PD functional theory, we take the empirical approach along the lines of the previous extension theories of the DFT. Judging from the present results that are obtained by using the RECCI-based KE functionals, it seems that the assumption in the PD functional theory would not cause a fatal problem for atomic systems.

[27] P. Hohenberg and W. Kohn, Phys. Rev. 136, B864 (1964).

[28] W. Kohn and L. J. Sham, Phys. Rev. 140, A1133 (1965).

[29] E. R. Davidson, Chem. Phys. Lett. 246, 209 (1995).

[30] S. Kh. Samvelyan, Int. J. Quantum Chem. 65, 127 (1997).

[31] M.-E. Pistol, Chem. Phys. Lett. 400, 548 (2004).

[32] P. W. Ayers and E. R. Davidson, Int. J. Quantum Chem. 106, 1487 (2006).

[33] P. Gori-Giorgi and A. Savin, Philos. Mag. 86, 2643 (2006).

[34] M.-E. Pistol, Chem. Phys. Lett. 417, 521 (2006).

[35] M.-E. Pistol, Chem. Phys. Lett. 422, 363 (2006).

[36] M.-E. Pistol, Chem. Phys. Lett. 431, 216 (2006).

[37] P. W. Ayers, Phys. Rev. A 74, 042502 (2006).

[38] P. W. Ayers and S. Liu, Phys. Rev. A 75, 022514 (2007).

[39] P. W. Ayers and E. R. Davidson, in Reduced-Density-Matrix Mechanics with Application to Many-Electron Atoms and Molecules, edited by D. A. Mazziotti, Advances in Chemical Physics Vol. 134 (Wiley, New York, 2007), p. 443.

[40] M.-E. Pistol, Chem. Phys. Lett. 449, 208 (2007).

[41] N. H. March and R. Santamaria, Int. J. Quantum Chem. 39, 585 (1991).

[42] P. W. Ayers, J. Math. Phys. 46, 062107 (2005).

[43] R. Cuevas-Saavedra and P. W. Ayers, Int. J. Quantum Chem. 109, 1699 (2009).

[44] D. Chakraborty and P. W. Ayers, J. Math. Chem. 49, 1810 (2011).

[45] K. Higuchi and M. Higuchi, Phys. Rev. A 85, 062508 (2012).

[46] G. Vignale and M. Rasolt, Phys. Rev. Lett. 59, 2360 (1987).

[47] G. Vignale and M. Rasolt, Phys. Rev. B 37, 10685 (1988).

[48] M. Higuchi and A. Hasegawa, J. Phys. Soc. Jpn. 66, 149 (1997).

[49] M. Higuchi and A. Hasegawa, J. Phys. Soc. Jpn. 67, 2037 (1998).

[50] J. P. Perdew and Y. Wang, Phys. Rev. B 33, 8800 (1986).

[51] J. P. Perdew, in Electronic Structure of Solids '91, edited by P. Ziesche and H. Eschrig (Akademie Verlag, Berlin, 1991), p. 11.

[52] J. P. Perdew, K. Burke, and M. Ernzerhof, Phys. Rev. Lett. 77, 3865 (1996).

[53] J. P. Perdew, A. Ruzsinszky, G. I. Csonka, O. A. Vydrov, G. E. Scuseria, L. A. Constantin, X. Zhou, and K. Burke, Phys. Rev. Lett. 100, 136406 (2008); 102, 039902(E) (2009).

[54] V. N. Staroverov, G. E. Scuseria, J. Tao, and J. P. Perdew, Phys. Rev. B 69, 075102 (2004).

[55] S. Liu and R. G. Parr, Phys. Rev. A 53, 2211 (1996).

[56] S. Liu, Á. Nagy, and R. G. Parr, Phys. Rev. A 59, 1131 (1999).
[57] Á. Nagy, S. B. Liu, and R. G. Parr, Phys. Rev. A 59, 3349 (1999).

[58] E. Bene and Á. Nagy, Chem. Phys. Lett. 324, 475 (2000).

[59] S. B. Liu, F. De Proft, Á. Nagy, and R. G. Parr, Adv. Quantum Chem. 36, 77 (2000).

[60] P. W. Ayers, J. B. Lucks, and R. G. Parr, Acta Chim. Phys. Debricina 34-35, 223 (2002).

[61] K. Higuchi and M. Higuchi, Phys. Rev. B 74, 195122 (2006); 75, 159902(E) (2007).

[62] M. Higuchi and K. Higuchi, Phys. Rev. B 75, 195114 (2007).

[63] K. Higuchi and M. Higuchi, J. Phys.: Condens. Matter 19, 365216 (2007).

[64] M. Higuchi and K. Higuchi, Phys. Rev. A 81, 042505 (2010).

[65] O. Gunnarsson and P. Johansson, Int. J. Quantum Chem. 10, 307 (1976).

[66] O. Gunnarsson, M. Jonson, and B. I. Lundqvist, Solid State Commun. 24, 765 (1977).

[67] O. Gunnarsson, M. Jonson, and B. I. Lundqvist, Phys. Rev. B 20, 3136 (1979).

[68] O. Gunnarson and R. O. Jones, Phys. Scr. 21, 394 (1980).

[69] J. A. Alonso and L. A. Girifalco, Solid State Commun. 24, 135 (1977).

[70] J. A. Alonso and L. A. Girifalco, Phys. Rev. B 17, 3735 (1978).

[71] M. Higuchi and K. Higuchi, Phys. Rev. B 65, 195122 (2002).

[72] L. H. Thomas, Proc. Cambridge Philos. Soc. 23, 542 (1927).

[73] E. Fermi, Rend. Accad. Lincei 6, 602 (1927).

[74] R. G. Parr and W. Yang, Density-Functional Theory of Atoms and Molecules (Oxford University Press, New York, 1989), Chap. 6.

[75] C. Lee and R. G. Parr, Phys. Rev. A 35, 2377 (1987).

[76] C. F. von Weizsäcker, Z. Phys. 96, 431 (1935).

[77] O. Gunnarsson and B. I. Lundqvist, Phys. Rev. B 13, 4274 (1976).

[78] M. Levy and J. P. Perdew, Phys. Rev. A 32, 2010 (1985).

[79] J. Wang and V. H. Smith Jr., Phys. Rev. A 52, 1060 (1995).

[80] S. J. Chakravorty, S. R. Gwaltney, E. R. Davidson, F. A. Parpia, and C. F. Fischer, Phys. Rev. A 47, 3649 (1993).

[81] E. R. Davidson, S. A. Hagstrom, S. J. Chakravorty, V. M. Umar, and C. F. Fischer, Phys. Rev. A 44, 7071 (1991).

[82] A. V. Bunge and R. O. Esquivel, Phys. Rev. A 34, 853 (1986).

[83] M. A. Buijse and E. J. Baerends, in Density Functional Theory of Molecules, Clusters, and Solids, edited by D. E. Ellis (Kluwer Academic, Dordrecht, 1995), p. 1.

[84] The scaling property of the KE of the PD functional theory is given by $T\left[\gamma_{\zeta}^{(2)}\right]=\zeta^{2} T\left[\gamma^{(2)}\right]$ [9]. Note that this scaling property is different from the scaling property of the KE functional of the DFT [78].

[85] G. D. Mahan, Many-Particle Physics (Kluwer Academic/ Plenum, New York, 2000), Chap. 5.

[86] Concerning the exceptionally small value of $\Delta T_{\text {functional }}$ for the TFW-LDA, we have already discussed this in Sec. IV A 1.

[87] S. Liu, Phys. Rev. A 54, 4863 (1996).

[88] S. Liu, V. Karasiev, R. L. Boada, and F. de Proft, Int. J. Quantum Chem. 69, 513 (1998).

[89] S. Liu and R. G. Parr, J. Phys. Chem. A 111, 10422 (2007). 